\title{
BRIGHT ASTEROIDS IN THE RED SEA - AN EMERGING PATHOGEN CANDIDA HAEMOLUNII: ARE AZOLES THE TREATMENT OPTION?
}

\author{
SANYUKTHA T ${ }^{1}$, POOJA RAO ${ }^{2 *}$, KEERTHIRAJ B ${ }^{3}$, RADHAKRISHNA M $^{1}$
}

${ }^{1}$ Department of Microbiology, Kasturba Medical College, Manipal University, Mangaluru, Karnataka, India. ${ }^{2}$ Department of Microbiology, Kasturba Medical College, Manipal University, Mangaluru, Karnataka, India. ${ }^{3}$ Department of Radiology, Kasturba Medical College, Manipal University, Mangaluru, Karnataka, India Email: poojakeerthiraj@yahoo.co.in

Received: 20 October 2016, Revised and Accepted: 02 November 2016

ABSTRACT

This is a case study of a 15-year-old female who on chemotherapy and central line catheter-related bloodstream candidemia presented with recurrent episodes of candidemia, was febrile and breathless. She was diagnosed of Candida haemulonii fungemia. Echinocandins was started as this species of Candida is usually resistant to the commonly used antifungal agents. On treatment, the patient was afebrile and discharged.

Keywords: Candida haemulonii, Candidemia, Case report, Emerging pathogen

(c) 2017 The Authors. Published by Innovare Academic Sciences Pvt Ltd. This is an open access article under the CC BY license (http://creativecommons. org/licenses/by/4. 0/) DOI: http://dx.doi.org/10.22159/ajpcr.2017.v10i2.15775

\section{INTRODUCTION}

Candidemia is one of the frequently seen blood stream infections. Candida haemulonii, one of the non-albicans Candida species, is known to be resistant to amphotericin B and azoles [1]. It is an emerging yeast pathogen involved in human infections such as onychia, ulcers of the feet or legs, and candidemia [2]. The treatment regimen for invasive $C$. haemulonii infections has not been clearly established. We investigated a case of febrile neutropenia catheter-related blood stream infection with recurrent episodes of candidemia due to $C$. haemulonii.

\section{CASE REPORT}

A 15-year-old female, a known case of osteosarcoma, was admitted for chemotherapy on June 3,2015, in the department of pediatric oncology and had undergone left limb salvage surgery in the past. In the following $5^{\text {th }}$ day of chemotherapy, she had dehydration, so intravenous fluids were administered, and the very next day, fever spikes were seen. Blood culture report revealed no growth. She was discharged on June 30, 2015.

The patient was readmitted on July 13, 2015 for chemotherapy, and on the $3^{\text {rd }}$ day, the patient showed dehydration and central line catheter was inserted. On July 23, 2015, the patient showed visible symptoms of fever, cold, cough, chest pain, palpitations, and breathlessness. Blood culture and Gram stain revealed Candida, hence azoles were started. The final blood culture report revealed $C$. haemulonii and second blood culture report revealed Candida parapsilosis. Central line catheter was removed immediately. This case revealed febrile neutropenia catheterrelated blood stream infection due to recurrent episodes of candidemia. As azoles and amphotericin B were found to be resistant in most of the C. haemulonii infections, micafungin belonging to echinocandins and flucytosine being nucleoside analog was given for 10 days and the patient was afebrile on June 30, 2015. On August 17, 2015, chemo port was removed and she was discharged.

The patient's consent and Institutional Ethical Committee clearance were obtained. Blood cultures were routinely collected in aerobic media and incubated using the BacT/ALERT system (bioMerieux, USA). Cultures with a positive signal in the microbial automated detection system (i.e., alarm-positive cultures) were subcultured by standard methods onto blood agar, chocolate agar, and MacConkey's medium, and aliquot was taken from positive bottles for Gram stain. Identification and antimicrobial susceptibility testing of the isolates to antimicrobial agents was performed using the VITEK2 system, bioMerieux, France. The presumptive identification of Candida isolates was done by conventional methods such as colony morphology showing white-tocream-colored pasty colonies, Gram staining from the colony showed Gram-positive budding yeast cells with or without pseudohyphae, germ tube test being negative, and HICHROME differential medium. The former sample was identified as C. haemulonii after $19 \mathrm{hrs}$ of incubation with resistance to amphotericin B with minimum inhibitory concentration (MIC) $\geq 16$ and azoles such as fluconazole with MIC $\geq 64$ and voriconazole with $\mathrm{MIC} \geq 8$ and sensitive to echinocandins such as caspofungin with $\mathrm{MIC}=0.5$, micafungin with $\mathrm{MIC}=0.25$, and flucytosine $\mathrm{MIC}=64$. The latter sample was identified as $C$. parapsilosis after 31 hrs of incubation and was sensitive to all the antifungal drugs, i.e., amphotericin B with $\mathrm{MIC}=0.5$, fluconazole with $\mathrm{MIC} \leq 1$, voriconazole with $\mathrm{MIC} \leq 1$, flucytosine with $\mathrm{MIC} \leq 1$, and caspofungin and micafungin with $\mathrm{MIC}=0.5$.

A study was done to check the biofilm production by crystal violet assay and antifungal susceptibility for azoles by Kirby-Bauer's method. It was performed on Mueller-Hinton agar supplemented with $2 \%$ glucose and $0.5 \mu \mathrm{g} / \mathrm{ml}$ of methylene blue for enhancing the definition of growth margins [3]. C. parapsilosis showed weak adherence and was sensitive to both the antifungal agents $(20 \mathrm{~mm})$ while $C$. haemulonii revealed strong biofilm production and was resistance to fluconazole (zone diameter $=6 \mathrm{~mm}$ ) and sensitive to voriconazole (zone diameter $=27 \mathrm{~mm}$ ). When compared with VITEK MIC results, voriconazole with MIC $\geq 8$ was resistant which indicates very major error (VME), this is due to false-negative disk diffusion results and is usually seen in non-Candida albicans species [4]. In such cases, VITEK (MIC) results should be considered (MIC interpretive criteria for voriconazole were those approved by the Clinical and Laboratory Standards Institute [CLSI] as follows: Susceptible, MIC of $\leq 1 \mu \mathrm{g} / \mathrm{ml}$; susceptible dose dependent, MIC of $2 \mu \mathrm{g} / \mathrm{ml}$; and resistant, MIC of $\geq 4 \mu \mathrm{g} / \mathrm{ml}$. The interpretive criteria for the voriconazole disk diffusion method were those used by the CLSI [CLSI document M44-A, 2005], as follows: Susceptible, zone diameter of $\geq 17 \mathrm{~mm}$; susceptible dose dependent, zone diameter of 14 to $16 \mathrm{~mm}$; and resistant, zone diameter of $\leq 13 \mathrm{~mm}$ ). MEs were classified as resistant by disk diffusion (participant or central laboratory) and susceptible by broth microdilution (BMD) or resistant by the participant disk diffusion test and susceptible by the central laboratory disk diffusion test. VMEs were classified as susceptible by 
the participant or central laboratory disk diffusion test and resistant by BMD or susceptible by the participant disk diffusion test and resistant by the central laboratory disk diffusion test. MEs occurred when the result of one of the tests was susceptible or resistant and that of the other test was susceptible dose dependent [4].

\section{DISCUSSION}

Previous reports and our case show that $C$. haemulonii is susceptible to echinocandins such as caspofungin or micafungin [1] and is resistant to amphotericin $\mathrm{B}$ and fluconazole $[1,2]$. There are only few cases that reveal the eradication of $C$. haemulonii-related candidemia through echinocandins administration $[1,5,6]$. In some cases, the administration of azoles such as fluconazole has failed to eradicate candidemia $[5,6]$. Identical patterns of susceptibility, with borderline sensitivity to amphotericin B, dose-dependent sensitivity to fluconazole, and resistance to the echinocandins tested (micafungin) were seen in 3 cases [7]. VME is rare and if it occurs, it is usually seen in non-C. albicans species. Risk factors for candidemia are the use of central venous catheters, total parenteral nutrition, use of multiple antibiotics, previous steroid therapy, postsurgery, and an immunocompromised status $[8,9]$. In our case study, long-term central venous catheterization for hyperalimentation and chemotherapeutic agents was the associated risk factor and no other source of infection was obtained. In previous case reports, the majority of patients received an intravenous central line and were in an immunocompromised state [1].

\section{CONCLUSION}

C. haemulonii is considered to be an emerging yeast pathogen for which the approach of patient management has yet to be illuminated. This is the first case to be reported in Dakshina Kannada. Fluconazole and amphotericin B are not a drug of choice for the treatment of $C$. haemulonii candidemia. Echinocandins, such as caspofungin or micafungin, may be an appropriate empirical choice of antifungal agent for invasive
C. haemulonii infections. This case illustrates the importance of correct identification of Candida species and the use of appropriate drug for the treatment.

\section{REFERENCES}

1. Kim S, Ko KS, Moon SY, Lee MS, Lee MY, Son JS. Catheter-related candidemia caused by Candida haemulonii in a patient in long-term hospital care. J Korean Med Sci 2011;26(2):297-300.

2. Rodero L, Cuenca-Estrella M, Córdoba S, Cahn P, Davel G, Kaufman S, et al. Transient fungemia caused by an amphotericin B-resistant isolate of Candida haemulonii. J Clin Microbiol 2002;40(6):2266-9.

3. Matar MJ, Ostrosky-Zeichner L, Paetznick VL, Rodriguez JR, Chen E, Rex JH. Correlation between E-test, disk diffusion, and microdilution methods for antifungal susceptibility testing of fluconazole and voriconazole. Antimicrob Agents Chemother 2003;47(5):1647-51.

4. Pfaller MA, Boyken L, Messer SA, Tendolkar S, Hollis RJ, Diekema DJ. Comparison of results of voriconazole disk diffusion testing for Candida species with results from a central reference laboratory in the ARTEMIS global antifungal surveillance program. J Clin Microbiol 2005;43(10):5208-13.

5. Khan ZU, Al-Sweih NA, Ahmad S, Al-Kazemi N, Khan S, Joseph L, et al. Outbreak of fungemia among neonates caused by Candida haemulonii resistant to amphotericin $\mathrm{B}$, itraconazole, and fluconazole. J Clin Microbiol 2007;45(6):2025-7.

6. Ruan SY, Kuo YW, Huang CT, Hsiue HC, Hsueh PR. Infections due to Candida haemulonii: Species identification, antifungal susceptibility and outcomes. Int J Antimicrob Agents 2010;35(1):85-8.

7. Muro MD, Motta Fde A, Burger M, Melo AS, Dalla-Costa LM. Echinocandin resistance in two Candida haemulonii isolates from pediatric patients. J Clin Microbiol 2012;50(11):3783-5

8. Hong SK, Nam SH, Kim HC. Fatal peripheral candidal suppurative thrombophlebitis in a postoperative patient. J Korean Med Sci 2008;23(6):1094-6.

9. Han SS, Yim JJ, Yoo CG, Kim YW, Han SK, Shim YS, et al. Clinical characteristics and risk factors for nosocomial candidemia in medical intensive care units: Experience in a single hospital in Korea for 6.6 years. J Korean Med Sci 2010;25(5):671-6. 